\title{
Research on the Complexity of Forms and Structures of Urban Green Spaces Based on Fractal Models
}

\author{
Yilu Gong, ${ }^{1,2}$ Xueming Li ${ }^{1},{ }^{1}$ Xueping Cong ${ }^{(D},{ }^{1}$ and He Liu ${ }^{1}$ \\ ${ }^{1}$ Geographical Sciences School, Liaoning Normal University, Dalian 116029, China \\ ${ }^{2}$ Art and Design School, Dalian Polytechnic University, Dalian 116034, China \\ Correspondence should be addressed to Xueming Li; lixueming999@163.com
}

Received 5 May 2020; Revised 27 May 2020; Accepted 1 June 2020; Published 24 July 2020

Guest Editor: Wen-Ze Yue

Copyright (c) 2020 Yilu Gong et al. This is an open access article distributed under the Creative Commons Attribution License, which permits unrestricted use, distribution, and reproduction in any medium, provided the original work is properly cited.

The fractal nature of urban green spaces is the product of the self-organizing evolution of the complex urban system into a higher stage, and orderly patterns and complex structures of urban green spaces will tend to manifest after they develop to a certain stage. On the basis of GF1 satellite data in 2019 and three fractal models, the complexity of the forms and structures of a green space system in downtown Dalian, China, was studied. The results showed that the boundary dimension measured by the perimeter-scale model was $0.64-1.40$, and the boundary dimension measured by the area-perimeter model was 1.79-1.99; these results indicate that the degree of human disturbance in green space boundaries was high, and the stability of the green space spatial structures was poor. The grid dimension measured by the area-scale model was $0.49-1.42$, and the average radius dimension measured by the area-radius model was $0.35-0.76$; these results indicate that the balance of the spatial distribution of green spaces was low, and the green spaces were excessively concentrated in the city center. Through comparisons of the scaling range among various types of green spaces, the characteristic range (gradient structure) of the spatial distribution of urban green spaces was found, which can reflect the maturity of green space growth and the quality of the green space layout. The gradient structure of green spaces in Dalian was characterized by three gradients, namely, 0-4000 m, 4000-8000 m, and 8000-16,000 m. The development of green spaces in the first gradient zone was the best, and the second and third gradient zones showed relatively large potential for improvement. These research results are of practical significance for guiding the planning and construction of green spaces in urban areas.

\section{Introduction}

Complexity is widely recognized by the scientific community as an important subject, and advanced understanding of spatial complexity has originated from studies on fractal cities. A fractal city is a research field that simulates and models cities based on fractal theory. The research on fractal cities began in the West in the early 1980s and in China in the early 1990s. The developments in regard to fractal cities have so far involved the following three levels: the microlevel of fractals in urban architecture [1], the mesolevel of fractals in urban forms [2], and the macrolevel of fractals in urban systems $[3,4]$. A fractal is an optimized structure of nature and an effective tool for delineating spatial complexity [5]. Research on fractal cities is beneficial to the effective utilization of urban geographic space [6].
The analysis of urban forms and structures can reveal important geographic information on urban spatial development and regional urbanization. The fractals of urban forms based on fractal theory have attracted extensive attention, and numerous relevant research projects have been carried out in China and other countries. The fractal dimension is an effective measure of fractals, as it can condense numerous geospatial data into a simple number to reveal the spatiotemporal information hidden behind the city [7]. There are three types of fractal models, which represent different geographic and geometric meanings. The boundary dimension can measure the complexity of the form of boundary lines in a city or for certain types of land use [8]. The radius dimension can describe the degree of concentration in the land use distribution [9]. The grid dimension (box dimension) is the most widely applied metric in various 
disciplines, and it can measure the balance of the spatial distribution in urban land use [10]. Additionally, Chen and Liu [11] developed the information dimension based on the grid dimension, and Qin et al. [12] developed a three-dimensional fractal approach based on two-dimensional fractals. Through fractal simulations and fractal dimension measurements of a large number of cities, Batty [13] found that the cities whose empirical values of urban form dimension were between 1 and 2 had an average dimension of 1.71. Chen and Luo [14] proposed the "theoretical interpretation of the average fractal dimension value" and stated that the "ideal urban fractal dimension evolves towards 1.71"; further, Chen and Luo [14] proposed that "the overall urban fractal dimension is not greater than the spatial dimension 2 where it is located, while the fractal dimension of various types of land use is not restricted." The above classic theories and typical practices related to urban fractal forms have important reference value for obtaining a thorough understanding of the complexity of urban spaces.

The urban fractal form is an orderly pattern and complex structure that emerges at a certain stage of urban development, and the fractal form of a certain type of urban land use is a product of the self-organizing evolution of the urban system and its internal subsystems to a higher stage. The urban green space fractals are separated from the urban fractal forms, and urban green space fractal research has gradually developed into a new branch. Urban green spaces play significant roles in improving heat island effects [15], land surface temperature [16], and urban wind and thermal environment [17-19]. Further, urban green spaces have economic and aesthetic values [20] and are an important part of green development, urban construction, and human living environments. Thus, research on urban green space fractals is of great significance for the spatial development of green spaces.

Batty and Longley [21] initially regarded green spaces as a part of "open spaces" in the study on the land use structure of Swindon, England, and their boundary dimension was calculated as 1.08. Zhao et al. [22] counted green spaces as "other lands" when studying the land use structure of Shanghai, and their boundary dimension was calculated as 1.11 , while the radius dimension was calculated as 1.78 . Zhao et al. [23] calculated that the green spaces in Shenyang City had a boundary dimension of 1.32 , a radius dimension of 2.64, and a grid dimension of 1.32. Chen et al. [24] chose "public green spaces" among green spaces when studying the fractal nature of land use forms in Changsha City, whose radius dimension was $0.8-1.0$. Zhu and $\mathrm{Li}$ [25] studied the multiscale transformation of the land use structure fractals in Liaoning Province and counted green spaces as three types, namely, "forest land," "grassland," and "cultivated land"; their boundary dimensions were calculated to be between 1.43 and 1.78. Yu and Yuan [26] used a fractal model to specifically calculate the spatial pattern of "green spaces for parks" in Fuzhou City, and a radius dimension of 1.23 was obtained, as well as a grid dimension of 1.27. Petrişor et al. [27] studied the overall fractal nature of "green infrastructure" in 14 cities in Romania. They divided the green infrastructure into agricultural landscapes, greenspace-forests, sport and leisure facilities, and water, and the fractal dimension results were between 0.30 and 0.90 . Jing et al. [28] took "recreational green spaces" as the research object and calculated the boundary dimension as 1.49-1.89 and the radius dimension as $0.79-1.11$. Liu et al. [29] measured the overall fractal dimension of the green space system in downtown Shanghai for many years; the average boundary dimension was 1.78 , and the average radius dimension was 3.04. Versini et al. [30] calculated the fractal dimensions of green roofs in nine European cities, and grid dimensions of $0.49-1.35$ were obtained.

Because the statistical calibers of urban green spaces in China and other countries are not uniform and different statistical approaches to green spaces can directly affect the results of fractal dimensions, comparisons cannot be made among the available studies. At present, the research on urban green space fractals mostly involves one type of green space as the research object, and research projects on fractal cases that make up urban green spaces as a system are few. In the research on the fractal nature of green space systems, the number of longitudinal comparative studies on fractal dimensions in different years is large, but there is a lack of horizontal comparative research on fractal dimensions of multiple types of green spaces within the green space system. This study treated the urban green space system as the research object and Dalian City in China was used as an example. Then, three models of the boundary dimension, radius dimension, and grid dimension were used to investigate the complexity of the overall form and structure of the green space system and the development characteristics of various types of green spaces. The innovative research value of this paper lies in the use of high-resolution remote sensing data to discuss the classification and classification criteria of green space. The study compares the three types of fractal models and the fractal features of each type of green space and puts forward scientific guidance and suggestions for urban green space construction.

\section{Research Data and Methods}

2.1. Overview of the Study Area. The downtown area of Dalian $\left(38^{\circ} 47^{\prime} \mathrm{N}-39^{\circ} 07^{\prime} \mathrm{N}, 121^{\circ} 16^{\prime} \mathrm{E}-121^{\circ} 45^{\prime} \mathrm{E}\right)$ is located at the southern tip of the Liaodong Peninsula on the shores of the Yellow Sea and Bohai Sea (Figure 1). Dalian is an important economic, trade, and port city on the east coast of China, with four administrative districts, namely, Zhongshan District, Shahekou District, Xigang District, and Ganjingzi District. The landforms of this area belong to those of a hilly and mountainous peninsula, and it has a temperate semihumid continental monsoon climate. Blessed by the unique landforms and climate characteristics, this area has become an international tourist city, famous for its urban green spaces. The urban green space system is mature and is characterized by fractal features and all types of green spaces, having a representative value in this study.

\subsection{Green Space Classification and Classification Criteria.} The green space system in downtown Dalian was reclassified based on the GF1 data in 2019, with the support of software, 


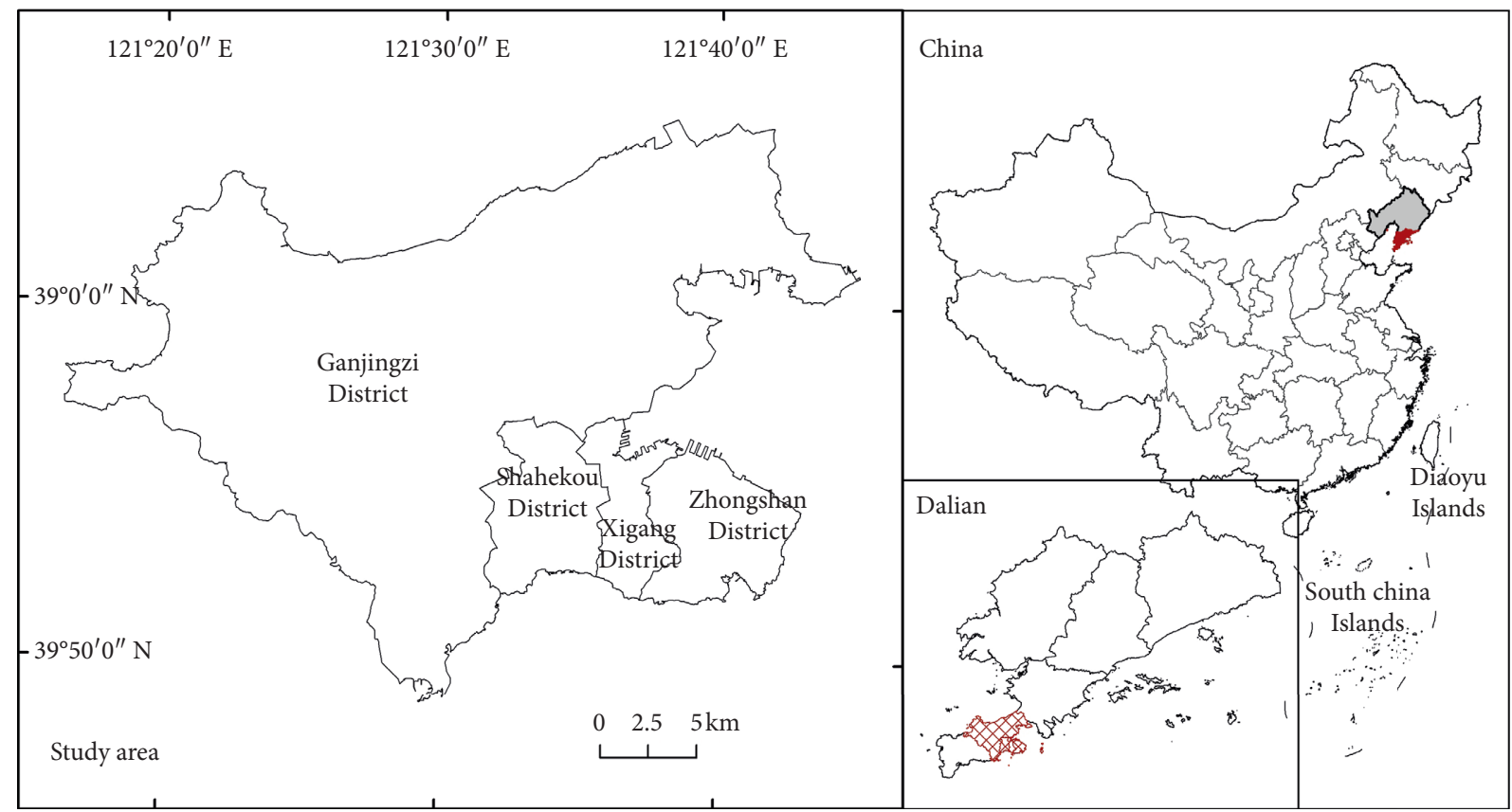

Figure 1: Location of the study area.

such as ENVI, ArcGIS, and Fragstasts4.3, and by using remote sensing interpretations, manual classifications, and field survey methods, in accordance with the "Urban Green Space Classification Standards" (CJJ/T85-2017) and the "Land Use Status Classification" (GBT21010-2017); furthermore, the reclassification was performed by taking into account the characteristics of green space vegetation in the study area. The results are listed in Table 1.

Urban green space classification is the technical foundation of urban planning, construction, and statistical management of green spaces by cities. The classification of green spaces in China is mostly based on the classification criteria for the land use status or urban green space classification standards. The land use classification focuses on the types and functions of vegetation itself, while urban green space classification pays more attention to the functions and forms of artificial green spaces. Urban centers have a high density of human settlements, and the forms and functions of artificial green spaces are more prominent. Therefore, the urban green space classification standards were selected as the main basis. However, when classifying according to the urban green space classification standards, not all types of woodlands can be counted, but here the number of woodland patches that could not be counted in the study area was too large to be ignored. Therefore, the classification criteria for land use status were taken as a secondary basis, and the "regional green spaces" and "woodlands" were combined and counted as "woodlands." The distribution map of reclassified green spaces is shown in Figure 2.

\subsection{Fractal Models}

2.3.1. Grid Dimension. The grid dimension is measured by using the box-counting method. A rectangular area is used to cover an urban geographic object in the map, such as an urban green space, and the length of one side of the rectangle is set as $r=L$. At this time, only one grid is occupied by the research object; that is, the number of nonempty grids is $N(L)=1$. Then, each side of the rectangular frame is divided into two equal segments, so the rectangle is divided into four congruent grids. Now the side of the small rectangle is $r=L / 2$, and the number of nonempty grids occupied by urban green spaces $N(L / 2)$ is counted. Furthermore, each side of the four small grids is divided into two equal segments, so the rectangular area is divided into 16 congruent parts, and the scale of each small rectangle is $r=L / 4=L / 2^{2}$; the number of nonempty grids occupied by the urban green space is $N\left(L / 2^{n}\right)$. If the urban green space is fractal, then, according to fractal theory,

$$
N\left(\frac{L}{2^{n}}\right)=2^{-D} N\left(\frac{1}{2^{n+1}}\right) .
$$

This obviously conforms to the negative power function:

$$
N(r) \propto r^{-D} \text {. }
$$

If the scale invariance defined by the above formula is fulfilled, then $D$ in this formula is the fractal dimension. If the grid scale $r$ and the number of nonempty grids $N(r)$ follow a negative exponential relationship, it can be judged that a fractal exists in the urban green space. For the overall fractal of the city, the grid dimension is generally $1 \leq D \leq 2$, but each type of land use is not restricted by this. The geographic and geometric meaning of the grid dimension represents the filling degree of a certain type of land use in the city, that is, the balance of land use. A larger grid dimension is indicative of an evener distribution of urban green spaces. Conversely, a smaller grid dimension is indicative of a more concentrated distribution of urban green spaces. 
TABLE 1: Green land type classifications and contents.

\begin{tabular}{|c|c|c|}
\hline Number & Type & Content and scope \\
\hline G & Green space system & $\begin{array}{c}\text { Including green spaces for parks, protective green spaces, land for squares, auxiliary green spaces, and } \\
\text { woodlands }\end{array}$ \\
\hline$G 1$ & $\begin{array}{l}\text { Green spaces for } \\
\text { parks }\end{array}$ & $\begin{array}{l}\text { Open to the public, with recreation as the main function, with functions of ecology, landscape, culture, } \\
\text { education, and emergency risk avoidance, as well as green space with certain recreation and service } \\
\text { facilities }\end{array}$ \\
\hline G2 & $\begin{array}{c}\text { Protective green } \\
\text { spaces }\end{array}$ & $\begin{array}{l}\text { The land is independent and has the functions of sanitation, isolation, safety, and ecological protection and } \\
\text { it is not suitable for visitors to enter the green space. Mainly include sanitary isolation protection green } \\
\text { space, road and railway protection green space, high-voltage corridor protection green space, and public } \\
\text { facilities protection green space }\end{array}$ \\
\hline G3 & Land for squares & $\begin{array}{l}\text { For urban public event venues with functions such as recreation, commemoration, assembly, and risk } \\
\text { avoidance, the green area should be greater than or equal to } 35 \% \text {, and the square area with green area } \\
\text { greater than or equal to } 65 \% \text { should be included in the park green space }\end{array}$ \\
\hline G4 & $\begin{array}{l}\text { Auxiliary green } \\
\text { spaces }\end{array}$ & $\begin{array}{l}\text { Afforestation land attached to various types of urban construction land (except "green space and square } \\
\text { land") including residential land, public management and public service facility land, commercial service } \\
\text { facility land, industrial land, logistics warehouse land, road and transportation facility land, public facility } \\
\text { land, and other green lands }\end{array}$ \\
\hline G5 & Woodlands & $\begin{array}{l}\text { Refers to the land where trees, bamboos, and shrubs grow, including tree woodland, bamboo woodland, } \\
\text { mangrove land, forest swamp, shrub woodland, shrub marsh, and other woodlands; other woodlands } \\
\text { include sparse forest land, undeveloped forest land, plot land, nurseries and other woodlands }\end{array}$ \\
\hline
\end{tabular}

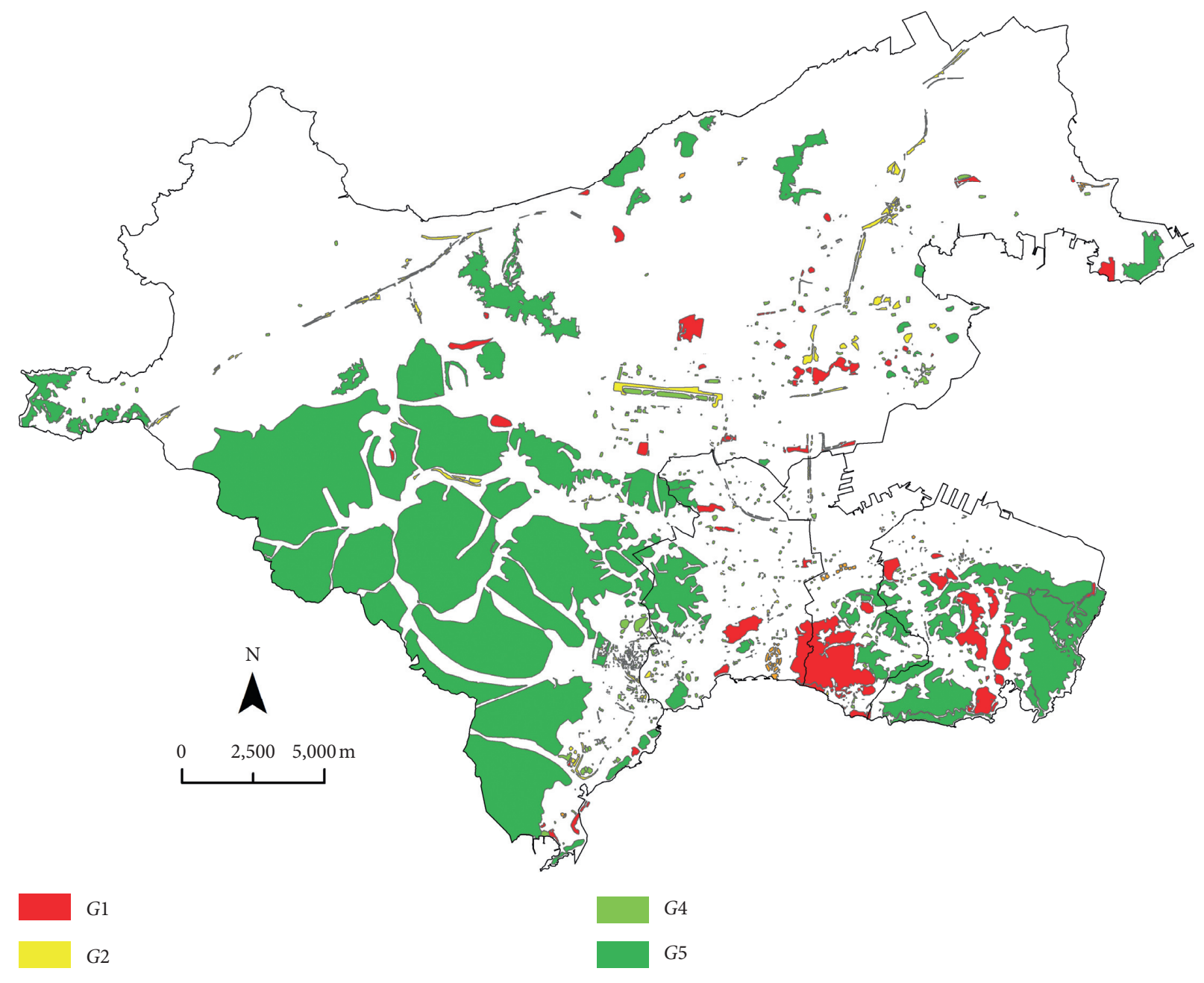

Figure 2: Distribution of green space types in the downtown area of Dalian. 
2.3.2. Boundary Dimension. There are two methods to define the boundary dimension. The fractal dimension defined based on the perimeter-scale relationship can be implemented by the box-counting method. According to fractal theory, the side of the rectangle $r$ and the number of nonempty grids covered by boundary lines of green spaces $N$ ( $r$ ) fulfill

$$
\ln N(r)=-D \ln r+C,
$$

where $C$ is a constant to be determined and $D$ is the boundary dimension.

The research idea of the fractal dimension defined based on the area-perimeter relationship is as follows. Assume that a certain type of land use in the city is a closed area, whose area is $A$ and the perimeter is $P$, and assume that the boundary is a fractal line represented by dimension $D$.

By using the box-counting method to estimate the fractal dimension, the number of nonempty grids covered by the boundary line $N(r)$ and the number of nonempty grids covered by the area $M(r)$ can be written as follows:

$$
\ln N(r)=\frac{2}{D} \ln M(r)+C .
$$

The boundary dimension defined by the perimeter scale only reflects the degree of complexity and sinuosity of the green space boundary line. A larger value of $D$ is indicative of a higher complexity of the boundary line, and, generally, $0<D<2$. The boundary dimension defined by the area perimeter can reflect more information. In addition to the complexity of the boundary line, it also reflects the degree of land fragmentation and the stability of land use structures [25]. The theoretical value of $D$ is between 1 and 2. When $D<1.50$, the form of urban green spaces tends to be simple; $D=1.50$ indicates that the form of green spaces is in a random state similar to Brownian motion; when $D>1.5$, the structure of urban green spaces is more complicated.

2.3.3. Radius Dimension. The radius dimension is a fractal dimension defined based on the law of distance attenuation, and it represents the urban center-edge distance attenuation relationship and reflects the urban growth characteristics. The radius method is used for the calculation. Assuming that concentric circles are made with the urban center as the center of the circles and the area $N(r)$ of a certain type of land use in the city within the circle with a radius $r$ is calculated, for fractal growth, there exists the following arearadius scaling relationship:

$$
\ln N(r)=\ln N_{0}+D \ln r,
$$

where $N_{0}$ represents a constant coefficient and $D$ is the radius dimension. The radius dimension represents the relative rate of density decay of a certain type of urban land use from the center to the edge. $D>2$ means that the density of urban green spaces increases from the center to the edge; $D=2$ means that the density of urban green spaces does not change; $D<2$ means that the density of urban green spaces decreases from the center to the edge.

\section{Results and Analysis}

3.1. Measurement of Grid Dimensions of Urban Green Spaces. First, it was determined whether the green spaces in downtown Dalian have fractal features. ArcGIS software was used to calculate the scale $r$ and the corresponding nonempty grid count $N(r)$. Taking $\ln r$ as the horizontal axis and $\ln N(r)$ as the vertical axis, the $\ln -\ln$ coordinate plot of all counted point ranges was constructed. The results showed that the point ranges had an obvious linear distribution trend. The power exponential model in equation (2) was applied to fit the points with the least square method, and the values of the goodness of fit coefficient $R^{2}$ were all above 0.99 . Preliminarily, the green spaces in downtown Dalian were judged to have fractal features.

Then, the scaling range was determined. Because the theoretical regular fractal does not exist in the real world, there must be a limited scaling range; that is, a strict straight line is rarely formed in the double logarithmic coordinate plot, but straight-line segments appear within certain intervals. For the fractal dimension of urban forms adopting the box method, the scaling range is usually easy to determine. The scaling range of the grid dimension of urban green spaces is discussed here for the comparison with the radius fractal dimension model, as shown in Figure 3.

Finally, the fractal dimension measurement was performed, and the confidence statement was given. The fractal dimension values of green spaces measured by the grid dimension model were between 0.59 and 1.42. Here, two groups of fractal dimension values, one with all point ranges involved in the calculation and the other with only the point ranges that were within the scaling range involved in the calculation, are listed for comparison. The key to the confidence statement lies in the significance level of the conclusion and the error range of the fractal dimension. By using the error range calculation method proposed by Chen [31], the calculation formula can be written as follows:

$$
D^{*}=D \pm \delta \times T_{\alpha,(n-2)},
$$

where $D^{*}$ represents the upper and lower bounds of the fractal dimension $\left(D_{u}, D_{1}\right), \delta$ is the standard error, $T_{\alpha,(n-2)}$ represents the threshold value of $T$ statistics, $\alpha$ is the significance level, and $n$ is the number of data point ranges used in the estimation of the fractal dimension. In this study, $\alpha=0.05$, and the fractal dimension results and confidence statements are listed in Table 2. Taking the urban green space system $G$ as an example, the results mean that we are 95\% confident that the fractal dimension of the green space system was 0.59 , and the error range was $0.58-0.60$.

The grid dimension represents the balance of the spatial distribution of urban green spaces. From the measurement results, it can be seen that, for the various types of green spaces in the green space system in Dalian, the order of balance was $G 3>G 2>G 4>G 4>G 1>G$. The balance of land for squares was the highest, as Dalian is famous for squares. The land patches for squares had small areas, large numbers, 


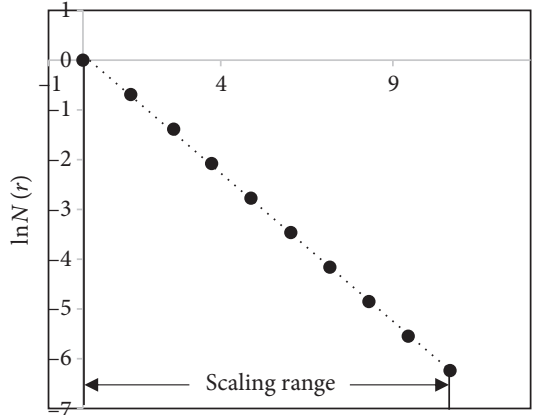

$\ln r$

(a)

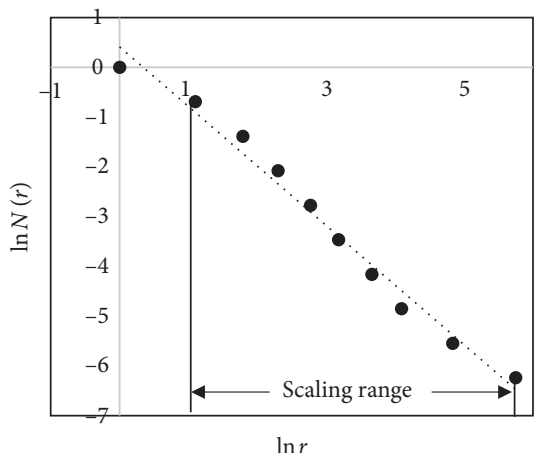

(d)

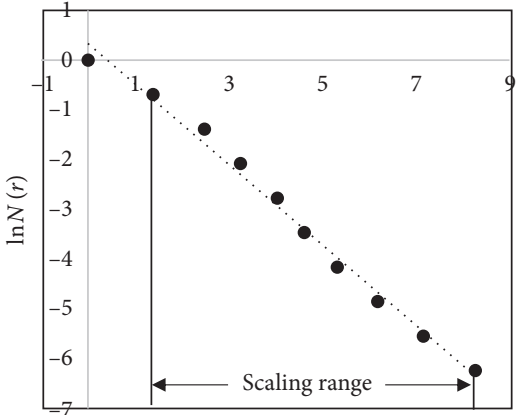

$\ln r$

(b)

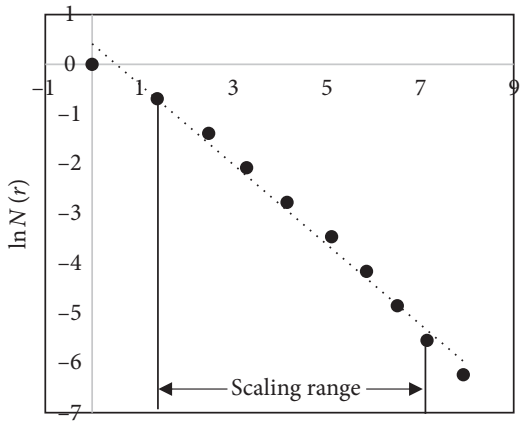

(e)

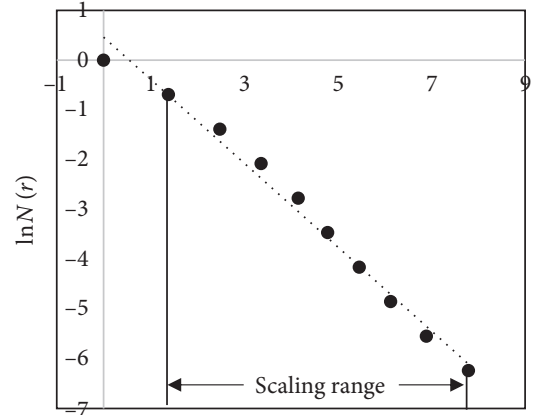

$\ln r$

(c)

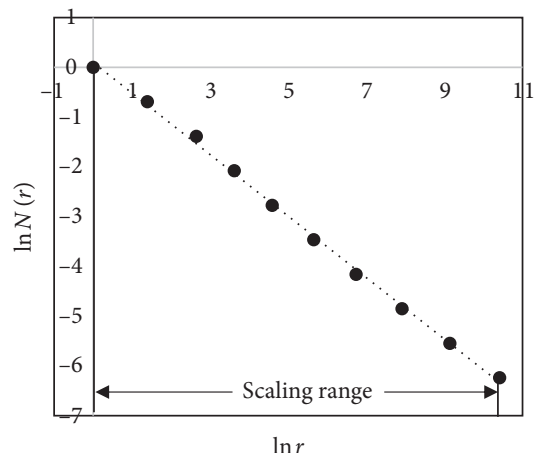

(f)

FIGURE 3: Bilogarithmic plots of various types of green space estimated by using grid dimensions: (a) G, (b) G1, (c) G2, (d) G3, (e) G4, and (f) G5.

TABLE 2: Fractal dimension results and confidence statements of various types of green space calculated by using the grid dimension.

\begin{tabular}{lccccccc}
\hline Range & Parameter & $G$ & $G 1$ & $G 2$ & $G 3$ & $G 4$ & G5 \\
\hline \multirow{4}{*}{ All points } & $D$ & 0.59 & 0.81 & 0.91 & 1.42 & 0.85 \\
& $R^{2}$ & 0.9995 & 0.9905 & 0.9947 & 0.9937 & 0.9944 & 0.9983 \\
& $\delta$ & 0.004 & 0.027 & 0.023 & 0.039 & 0.022 & 0.009 \\
& Tolerance scope & $0.58-0.60$ & $0.75-0.87$ & $0.86-0.96$ & $1.33-1.51$ & $0.80-0.90$ & $0.60-0.64$ \\
\hline \multirow{4}{*}{ Scaling range } & $D$ & 0.59 & 0.85 & 0.91 & 1.37 & 0.84 & 0.62 \\
& $R^{2}$ & 0.9995 & 0.9947 & 0.9947 & 0.994 & 0.994 & 0.9983 \\
& $\delta$ & 0.004 & 0.025 & 0.024 & 0.04 & 0.023 & 0.009 \\
& Tolerance scope & $0.58-0.60$ & $0.79-0.91$ & $0.85-0.97$ & $1.33-1.51$ & $0.79-0.89$ & $0.60-0.64$ \\
\hline
\end{tabular}

and a wide distribution, thus providing a variety of open spaces for public activities. The main parts of the protective green spaces were the protective green belts on both sides of urban roads, in which the fractal dimension reflected the balance of the urban traffic distribution to a certain extent, and the traffic fractal was the closest to the urban fractal [32]. The auxiliary green spaces had a low balance, and the possible reason for this was that the distribution of auxiliary lands was concentrated. The fractal dimension of the green spaces for parks was low, which was the result of multiple theme parks with large areas, such as forest parks, zoos, and botanical gardens, concentrated in Xigang District and Zhongshan District. The balance of woodlands was the lowest, which was consistent with the characteristic of the concentrated distribution of forest vegetation.
3.2. Measurement of Boundary Dimensions of Urban Green Spaces. The perimeter-scale relationship and area-perimeter relationship were used to measure the boundary dimensions of various types of green spaces in downtown Dalian. The measurement processes included preliminary judgment of whether the green spaces were fractal, determination of the scaling range, measurement of the fractal dimension, and formation of the confidence statement. The specific operation methods were consistent with those for the measurement of grid dimensions. The measurement results are listed in Table 3.

The results were as follows. (1) The urban green spaces showed obvious fractal features, and the confidence coefficient at a level of $95 \%$ was high. Compared with the urban boundary fractal, the boundary dimensions of green spaces 
TABLE 3: Fractal dimension results and confidence statements of various types of green spaces calculated by using the boundary dimension.

\begin{tabular}{lccccccc}
\hline Model & Parameter & $G$ & $G 1$ & $G 2$ & $G 3$ & $G 4$ & G5 \\
\hline \multirow{4}{*}{ Perimeter-scale } & $D$ & 0.64 & 0.91 & 0.91 & 1.40 & 0.83 & 0.69 \\
& $R^{2}$ & 0.9955 & 0.9967 & 0.9947 & 0.9902 & 0.9940 & 0.9950 \\
& $\delta$ & 0.015 & 0.018 & 0.023 & 0.069 & 0.026 & 0.017 \\
& Tolerance scope & $0.61-0.67$ & $0.87-0.95$ & $0.86-0.96$ & $1.21-1.59$ & $0.77-0.89$ & $0.66-0.72$ \\
\hline \multirow{4}{*}{ Area-perimeter } & $D$ & 1.86 & 1.90 & 1.99 & 1.99 & 1.99 & 1.79 \\
& $R^{2}$ & 0.9987 & 0.9974 & 1 & 1 & 1 & 0.9939 \\
& $\delta$ & 0.023 & 0.034 & 0 & 0 & 0.049 \\
& Tolerance scope & $1.81-1.91$ & $1.83-1.97$ & 1.99 & 1.99 & 1.99 & $1.68-1.90$ \\
\hline
\end{tabular}

measured by the perimeter-scale model were lower, while those measured by the area-perimeter model were too high. (2) The fractal dimensions measured by the perimeter-scale model were between 0.64 and 1.40 , and those measured by the area-perimeter model were between 1.79 and 1.99. The difference between these two was large, which indicates that the forms and structures of green spaces were more complex. The results also confirm that the geographic and geometric meanings of fractal dimensions represented by the two models were significantly different. (3) The perimeter-scale relationship model represented the complexity in the forms of green space boundaries, in which the order was $G 3>G 1=G 2>G 4>G 5>G$. The data also reflect the degrees of human disturbances in various types of green spaces, which were the highest in the square lands and the lowest in woodlands. (4) The area-perimeter relationship model could not reflect the complexity of the boundary lines in a simple manner, but the results were more accurate in terms of describing the degree of fragmentation and the instability of the green space structure. The $D$ values were all higher than 1.5 and close to 2, thus showing that the green space structures were more complex than a random distribution status, and the order was $G 2=G 3=G 4>$ $G 1>G>G 5$. In comparisons with the other three types of green spaces, the overall structure of the green space system and the structure of woodlands were relatively more rational.

\subsection{Measurement of Radius Dimensions of Urban Green}

Spaces. The measurement processes for the radius dimensions were as follows: (1) determine the location of the urban center, (2) determine the minimum radius and the scale, (3) the concentric circles centered at the urban center with determined radiuses and scales are drawn outwards until the whole research area is covered, (4) the green space area in each concentric circle is counted, (5) the area-radius double logarithmic coordinate diagram is plotted to preliminarily judge whether a fractal exists, and (6) the data are fitted with the model to determine the scaling range and calculate the radius dimension. The key to the measurement of radius dimensions of urban green spaces lies in the selection of the urban center, as well as the determination of the minimum radius, scale, and scaling range.

The selection of the urban center and the determinations of the minimum radius and scale are carried out as follows. Where the center of the circles is located directly affects the fractal dimension results. The center of the circles is usually chosen in three ways, namely, the center of gravity of the research area, the center of gravity of the urban green spaces, and the administrative center or the commercial center of the city. Only the appropriate scale selection can give effective estimation results of fractal dimensions with the corresponding measurement scale. The following two types of scales are usually selected: the arithmetic scale and the geometric scale. The center of the circles, the minimum radius, and the scale are determined with the ultimate goal of achieving the optimal coverage of the fractal dimension measurement. The comparison and analysis of the three selection schemes were as shown in Figure 4, and scheme (c) in the figure was determined as the optimal choice in this study.

The numbers of effective point ranges of different types of green spaces obtained from the calculation of schemes (a) and (b) in Figure 4 were too small, while scheme (c) provided more effective point ranges and also achieved relatively better coverage. The double logarithmic plot of each type of green space obtained by scheme (c) is shown in Figure 5. The results showed that, except for the land for squares, there were multiple straight-line segments, that is, scaling ranges, in the double logarithmic plots. It can be preliminarily judged that the green spaces in downtown Dalian had fractal features.

The determination of scaling ranges is carried out as follows. Compared with the box-counting method, the determination of scaling ranges when the fractal dimension is measured by the radius method is much more difficult. At present, there is no objective and universal method for determining scaling ranges. When the self-radioactivity and multiscale fractal properties exist at the same time, it is difficult to give a clear scaling range by means of the radius method. Therefore, the scaling range can only be determined through the goodness of fit $R^{2}$ of multiple fittings, where the scope of data point ranges is changed continuously. Other indicators included fractal dimension values $D$ and $\bar{D}$, values of the fit coefficient $R^{2}$, standard error $\delta$, and tolerance scope. The specific operation methods were consistent with those for the measurement of grid dimensions. The scaling range, fractal dimension result, and confidence statement of each type of green space are listed in Table 4.

The results were as follows.

(1) Except for the land for squares, the other types of green spaces all had significant fractal features. The 

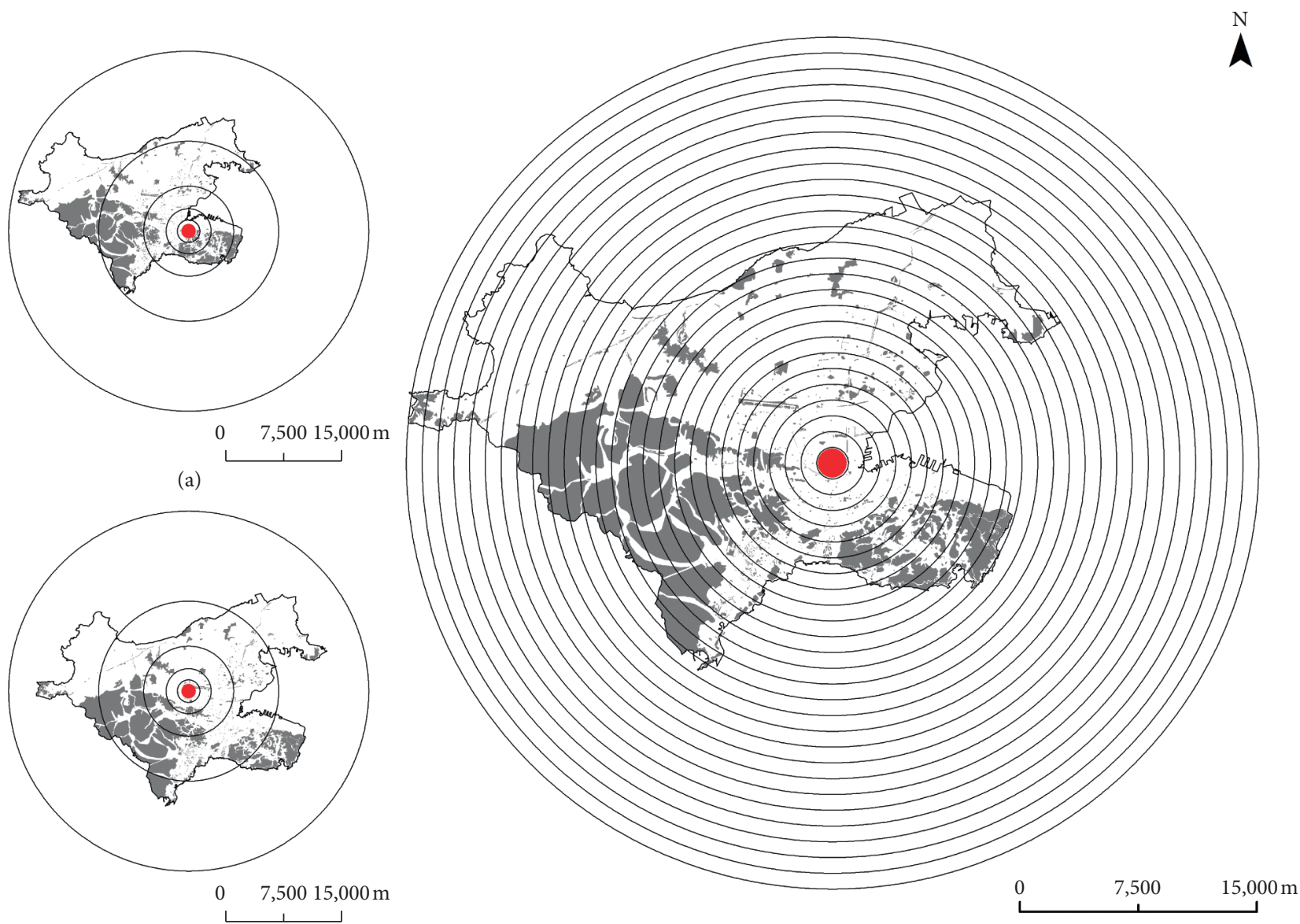

(b)

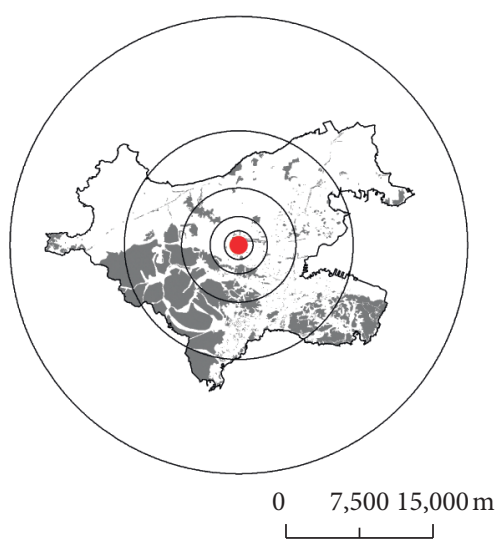

- Center of the circle

Green space

Concentric circles

(c)

FIgURE 4: Three schemes for the center, radius, and scale analysis. (a) Taking the city administrative center as the center, the geometric scale of radius $r=60,120,240, \ldots, 61,440 \mathrm{~m}$, a total of 10 sets of data points. (b) With the center of gravity of the study area as the center of the circle, the geometric scale of radius $r=25,50,100, \ldots, 51,200 \mathrm{~m}$, a total of 11 sets of data points. (c) Taking the center of urban green space (removing the woodland type) as the center of the circle, the radius $r=1000,2000,3000, \ldots, 27,000$, a total of 27 sets of data points.

auxiliary green spaces and woodlands showed single fractal characteristics, and the urban green space system and the green spaces for parks showed double fractal characteristics; meanwhile, the protective green spaces showed multifractal characteristics, which indicates that the spatial structure of the protective green spaces was the most complex.

(2) The number and scope of the scaling ranges had a characteristic significance. Judging from the comparison of the scaling ranges among various types of green spaces, the overall density distribution of the urban green space system showed "characteristic ranges," which are called the "gradient structure" in this study. The first gradient zone was $0-4000 \mathrm{~m}$, where the first scaling range of $G$, the first scaling range of G1, and G4 exhibited fractal features. The second gradient zone was $4000-8000 \mathrm{~m}$, where the second scaling range of $G$, the first scaling range of $G 2$, the second scaling range of $G 1$, and $G 5$ exhibited fractal features. The third gradient zone was $8000-16,000 \mathrm{~m}$, where the second scaling range of $G 2$, the third scaling range of $G 1$, and G5 showed fractal features. Due to the errors in the estimation of the scaling range using the radius method, the range of the gradient zone only represents a rough estimation. The gradient structure presented by the values is more important than the accuracy of the values themselves. When studying urban fractals, Zhang et al. [33] described that the first scaling range represents the urbanized area, and the second scaling range represents the area that may be urbanized in the future. The gradient structure described by the scaling range can reflect the maturity of urban green space growth and the quality of the green space layout. The length and location of the scaling range can reflect the range of the orderly distribution pattern of green spaces. It can be seen that the overall distribution pattern of the green space system in 


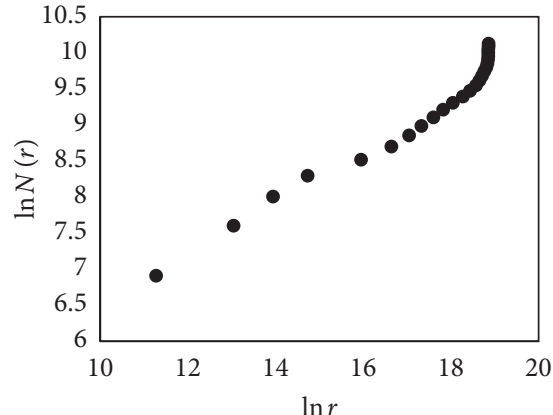

(a)

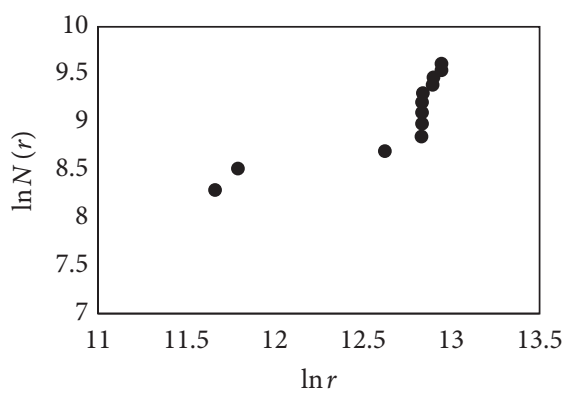

(d)

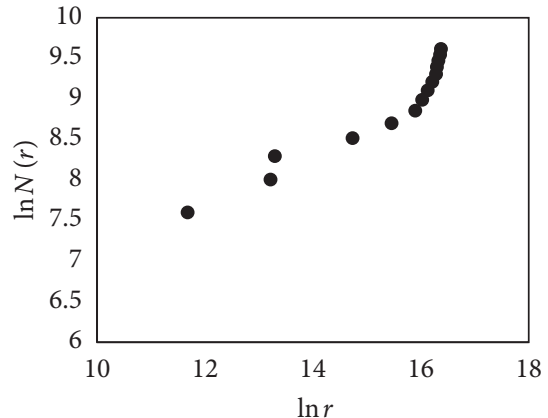

(b)

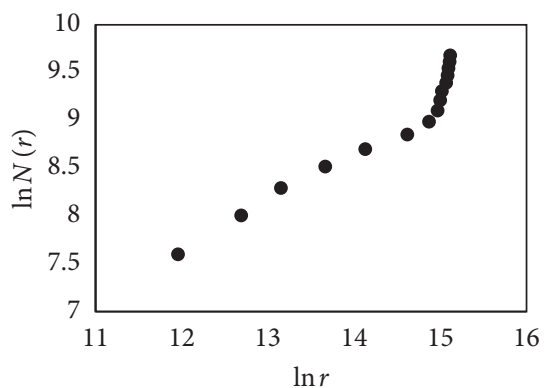

(e)

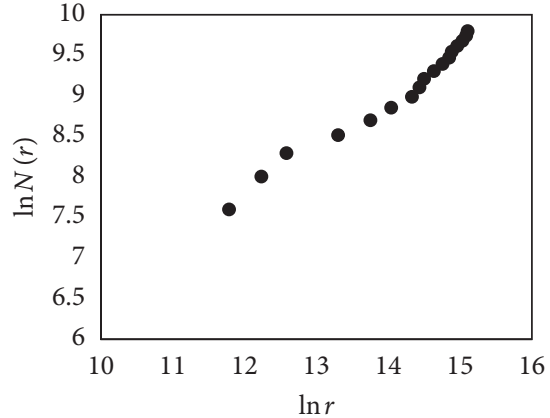

(c)

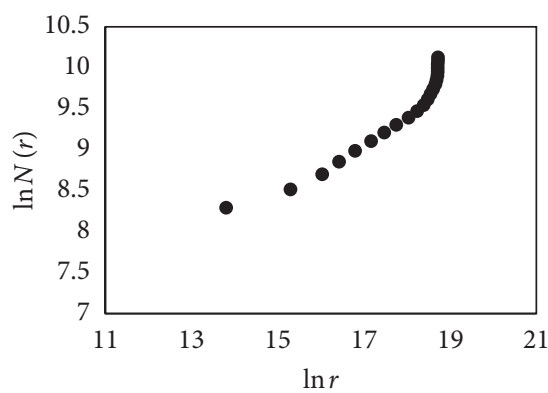

(f)

FIGURE 5: Bilogarithmic plots of various types of green space estimated by using the radius dimension. (a) G, (b) G1, (c) G2, (d) G3, (e) G4, and (f) G5.

TABLE 4: Scale areas, fractal dimension results, and confidence statements of various types of green land calculated by using the radius dimension.

\begin{tabular}{|c|c|c|c|c|c|c|c|c|c|}
\hline Parameter & \multicolumn{2}{|r|}{$G$} & \multicolumn{2}{|c|}{$G 1$} & \multicolumn{3}{|c|}{ G2 } & \multirow{2}{*}{$\begin{array}{c}\text { G4 } \\
\begin{array}{c}\text { Scaling } \\
\text { range }\end{array}\end{array}$} & \multirow{2}{*}{$\begin{array}{c}\text { G5 } \\
\begin{array}{c}\text { Scaling } \\
\text { range }\end{array}\end{array}$} \\
\hline $\begin{array}{l}\text { Scaling } \\
\text { range }(\mathrm{m})\end{array}$ & $\begin{array}{l}\text { First } \\
\text { scaling } \\
\text { range }\end{array}$ & $\begin{array}{c}\text { Second } \\
\text { scaling range }\end{array}$ & $\begin{array}{c}\text { First scaling } \\
\text { range }\end{array}$ & $\begin{array}{c}\text { Second } \\
\text { scaling range }\end{array}$ & $\begin{array}{c}\text { First scaling } \\
\text { range }\end{array}$ & $\begin{array}{l}\text { Second } \\
\text { scaling } \\
\text { range }\end{array}$ & $\begin{array}{c}\text { Third scaling } \\
\text { range }\end{array}$ & & \\
\hline & $0-4000$ & $4000-14000$ & $4000-7000$ & $7000-11000$ & $1000-4000$ & $4000-8000$ & $8000-17000$ & $0-5000$ & $4000-16000$ \\
\hline$D$ & 0.40 & 0.41 & 0.29 & 1.22 & 0.87 & 0.46 & 0.94 & 0.61 & 0.35 \\
\hline $\bar{D}$ & \multicolumn{2}{|c|}{0.40} & \multicolumn{2}{|c|}{0.76} & & 0.76 & & 0.61 & 0.35 \\
\hline$R^{2}$ & 0.9991 & 0.9911 & 0.9914 & 0.9979 & 0.9993 & 0.9969 & 0.991 & 0.9927 & 0.9917 \\
\hline$\delta$ & 0.008 & 0.013 & 0.026 & 0.039 & 0 & 0.018 & 0,033 & 0.03 & 0.01 \\
\hline $\begin{array}{l}\text { Tolerance } \\
\text { scope }\end{array}$ & $0.37-0.43$ & $0.38-0.44$ & $0-0.50$ & $1.05-1.39$ & 0.87 & $0.39-0.53$ & $0.97-1.01$ & $0.52-0.70$ & $0.33-0.37$ \\
\hline
\end{tabular}

downtown Dalian was orderly, and only the western and northeastern parts of Ganjingzi District did not have orderly characteristics. The lengths of the scaling range of green spaces for parks and the auxiliary green spaces were the shortest, thus indicating that there is large potential for improvement.

(3) From the fractal dimension results, it can be seen that the average fractal dimension values of different types of green spaces were between 0.35 and 0.76 , and the radius dimensions were all less than 2 , thus indicating that the decreasing rate of urban green spaces from the center to the periphery was rapid and that the green spaces were concentrated in the urban center. The fractal dimension of green spaces for parks increased by 0.93 in the two scaling ranges, which means that the distribution of green spaces for parks was too concentrated in the first scaling range. The fractal dimension of protective green spaces decreased first and then increased in the three scaling ranges, which was related to the functional characteristics of the protective green spaces themselves. There were large areas of traffic protective green spaces in the first scaling range and large areas of public health protective green spaces in the third scaling range.

\section{Discussion and Conclusions}

Fractal dimensions can measure fractals intuitively and effectively, and the different methods for fractal research pose an unavoidable scientific problem. Western scholars are good at measuring fractal dimensions by using computer 
simulation methods, while Chinese scholars focus more on mathematical models. In theory, when the measurement results of the three fractal models are closer, the urban fractals are simpler. Compared with an urban system, urban green space systems exhibit more complex fractal characteristics. For example, the results of boundary fractal dimensions measured by the area-perimeter model and the perimeter-scale model can vary greatly; additionally, the fractal dimension results measured by the area-perimeter model, area-scale model, and area-radius model are much smaller than the urban fractal dimension (1.71) [13], while the fractal dimension measured by the perimeter-scale model is much greater than the urban fractal dimension.

The technical problems of fractal dimension measurement and fractal judgment are difficulties in research on fractals. The scale, scaling range, standard error, goodness of fit, and confidence statement are all key parameters. The radius dimension model measures from bottom to top, and the measurement results are heavily dependent on the choice of the center of the circles. Therefore, Frankhauser and Sadler [9] called it the local fractal. Others also have discussed the importance of the center of the circles [31, 34, 35]. In this study, when studying the radius dimension of the urban green space, it was found that the center of the circles, the minimum radius, and the scale were three important parameters that interacted with each other and jointly affected the fractal dimension results. The complexity of different types of green spaces was manifested by the great difference in the locations of their respective centers of gravity. As a result, several types of green spaces had to be excluded. The green spaces for squares did not show fractal characteristics in the chosen scheme, which was not because the land for squares itself did not have a fractal nature; instead, the finding was related to the technical limitation of the radius dimension measurement.

Based on the GF1 data in 2019 and the three fractal models, the complexity of the forms and structures of the green space system and various types of green spaces in downtown Dalian was studied. The results showed that the grid dimension was $0.49-1.42$, the boundary dimension measured by the perimeter-scale model was $0.64-1.40$, the boundary dimension measured by the area-perimeter model was $1.79-1.99$, and the average radius dimension was $0.35-0.76$. These data demonstrate that the spatial forms and structures of the green space system in Dalian have a high complexity, which is manifested by the facts that the green space boundaries have a low sinuosity and high degree of human disturbance, the green space structures have low balance and poor stability, and the green space distribution is excessively concentrated in the urban center. The characteristic range (gradient structure) of the spatial distribution of the urban green space system was proposed through the scaling range, which can reflect the maturity of urban green space growth and the quality of the green space layout. The gradient structure of the green space in Dalian was found to be as follows: the first gradient zone is $0-4000 \mathrm{~m}$, the second gradient zone is $4000-8000 \mathrm{~m}$, and the third gradient zone is $8000-16000 \mathrm{~m}$.
The results of this study are of practical significance for improving the standards of construction and service of various types of urban green spaces. The specific suggestions for the planning and construction of the green space system in Dalian are to improve the balance of the green spaces for parks and the auxiliary green spaces and to integrate the protective green spaces, the land for squares, and the auxiliary green spaces, so as to reduce the fragmentation of green space patches. The area and number of green spaces for parks beyond $7000 \mathrm{~m}$ from the urban center and the area and number of auxiliary green spaces beyond $5000 \mathrm{~m}$ from the urban center ought to be increased, and their service level can be improved. The green space development within a circular area of $4000 \mathrm{~m}$ from the urban center is relatively orderly. The circular area with a radius of $4000-8000 \mathrm{~m}$ is the key area for recent green space development and construction, and the circular area with a radius of $8000-16,000 \mathrm{~m}$ is the key area for long-term green space development.

Additionally, the average value and the evolution process of the ideal value of the green space fractal dimensions have not yet been investigated. Therefore, it is necessary to carry out continuous research on the fractal nature of urban green spaces. Taking a large number of cities as cases and many years of high-resolution remote sensing data to study the average values and evolution of the fractal dimensions of various types of green spaces will be a new direction for the study of urban green space fractals in the future.

\section{Data Availability}

Data are available upon request to the corresponding author.

\section{Disclosure}

The funding sources had no role in the study design, data collection, analysis or interpretation, or the writing of this manuscript.

\section{Conflicts of Interest}

The authors declare that they have no conflicts of interest.

\section{Authors' Contributions}

Yilu Gong contributed to all aspects of this work. He Liu conducted the experiment and analyzed the data. Xueming $\mathrm{Li}$ and Xueping Cong revised the paper. All authors reviewed the manuscript.

\section{Acknowledgments}

This work was supported by the National Natural Science Foundation of China (Grant no. 41671158), Ministry of Education Humanities and Social Sciences Research Program (Grant no. 18YJCZH035), Liaoning Social Science Planning Fund Program (Grant no. L18CGL002), Liaoning Education Department Scientific Research Project (Grant no. J2020060), and Dalian Science and Technology Innovation Fund Project (Grant no. 2020JJ26GX039). In 
addition, the authors would like to thank Editage (https:// www.editage.cn) for the English language editing.

\section{References}

[1] C. Bovill, Fractal Geometry in Architecture and Design, Boston Birkhauser Publishing, Cambridge, MA, USA, 1996.

[2] B. H. Kaye, A Random Walk Through Fractal Dimensions, New York VCH Publishing, New York, NY, USA, 1989.

[3] S. L. Arlinghaus, "Fractals take a central place," Geografiska Annaler: Series B, Human Geography, vol. 67, no. 2, pp. 83-88, 1985.

[4] D. W. S. Wong and A. S. Fotheringham, "Urban systems as examples of bounded chaos: exploring the relationship between fractal dimension, rank-size, and rural to urban migration," Geografiska Annaler: Series B, Human Geography, vol. 72, no. 2-3, pp. 89-99, 1990.

[5] J. S. Liu and Y. G. Chen, "Cites, fractals and spatial complexity," Complex Systems and Complexity Science, vol. 3, pp. 62-69, 2004.

[6] Y. G. Chen, "Fractal cities and city planning," Urban Planning, vol. 2, pp. 33-40, 2005.

[7] Y. G. Chen and L. S. Huang, "Modeling growth curve of fractal dimension of urban form of Beijing," Physical A: Statistical Mechanics and Its Applications, vol. 523, pp. 1038-1056, 2019.

[8] M. Batty and P. A. Longley, "Fractal-based description of urban form," Environment \& Planning B: Planning and Design, vol. 14, no. 2, pp. 123-134, 1987.

[9] P. Frankhauser and R. Sadler, Fractal Analysis of Agglomerations, University of Stuttgart, Stuttgart, Germany, 1991.

[10] L. Benguigui, D. Czamanski, M. Marinov, and Y. Portugali, "When and where is a city fractal," Environment \& Planning B: Planning and Design, vol. 27, no. 4, pp. 507-519, 2000.

[11] Y. G. Chen and J. S. Liu, "An index of equilibrium of urban land-use structure and information dimension of urban form," Geographical Research, vol. 20, no. 2, pp. 146-152, 2001.

[12] J. Qin, C. L. Fang, Y. Wang, Q. Y. Li, and Y. J. Zhang, "A threedimensional box-counting method for estimating fractal dimension of urban form," Geographical Research, vol. 34, no. 1, pp. 85-96, 2015.

[13] M. Batty, "Cities as fractals: simulating growth and form," in Fractals and Chaos, pp. 43-69, Verlag Publishing, Berlin, Germany, 1991.

[14] Y. G. Chen and J. Luo, "A tentative theoretical interpretation of fractal dimension of urban form," Urban Studies, vol. 13, no. 5, pp. 35-40, 2006.

[15] A. Guo, J. Yang, X. Xiao, J. Xia, C. Jin, and X. Li, "Influences of urban spatial form on urban heat island effects at the community level in China," Sustainable Cities and Society, vol. 53, Article ID 101972, 2020.

[16] J. Yang, J. Sun, Q. S. Ge, and X. M. Li, “Assessing the impacts of urbanization-associated green space on urban land surface temperature: a case study of Dalian, China," Urban Forestry \& Urban Greening, vol. 22, pp. 1-10, 2017.

[17] J. Yang, Y. C. Wang, X. M. Xiao, C. Jin, J. C. Xia, and X. M. Li, "Spatial differentiation of urban wind and thermal environment in different grid sizes," Urban Climate, vol. 28, pp. 1-13, 2019.

[18] J. Yang, S. Jin, X. M. Xiao et al., "Local climate zone ventilation and urban land surface temperatures: towards a performancebased and wind-sensitive planning proposal in megacities," Sustainable Cities and Society, vol. 47, Article ID 101487, 2019.
[19] P. Xie, J. Yang, H. Wang, Y. Liu, and Y. Liu, "A new method of simulating urban ventilation corridors using circuit theory," Sustainable Cities and Society, vol. 59, Article ID 102162, 2020.

[20] J. Yang, Y. Y. Guan, J. C. Xia, C. Jing, and X. M. Li, "Spatiotemporal variation characteristics of green space ecosystem service value at urban fringes: a case study on Ganjingzi District in Dalian, China," Science of the Total Environment, vol. 639, pp. 1453-1461, 2018.

[21] M. Batty and P. A. Longley, "The morphology of urban land use," Environment and Planning B: Planning and Design, vol. 15, no. 4, pp. 461-488, 1988.

[22] J. Zhao, J.-H. Xu, and A.-X. Mei, "Fractal study on land use structure and form change: the case of central areas in Shanghai," Journal of East China Normal University (Natural Science), vol. 1, pp. 78-84, 2005.

[23] H. Zhao, D. M. Wang, and X. W. Tan, "A study on fractal characteristics of shenyang's urban form and spatial structure," Planners, vol. 2, no. 23, pp. 81-83, 2007.

[24] Q. Y. Chen, C. L. Yin, and G. H. Chen, "Spatial-temporal evolution of urban morphology and land use sorts in Changsha," Scientia Geographica Sinica, vol. 27, no. 2, pp. 273-280, 2007.

[25] X.-H. Zhu and Y.-Y. Li, "On multi-scale characteristics of structure of land use," Geographical Research, vol. 27, no. 6, pp. 1235-1242, 2008.

[26] S. J. Yu and S. Q. Yuan, "Study on the spatial pattern of urban public green space based on grids-a case study of the main urban area of Fuzhou," Journal of Fujian Normal University (Natural Science Edition), vol. 27, no. 6, pp. 88-94, 2011.

[27] A.-I. Petrişor, I. C. Andronache, L. E. Petrişor, A.-M. Ciobotaru, and D. Peptenatu, "Assessing the fragmentation of the green infrastructure in romanian cities using fractal models and numerical taxonomy," Procedia Environmental Sciences, vol. 32, pp. 110-123, 2016.

[28] Y. F. Jing, T. Li, C. C. Wang, and J. Shen, "Optimization of recreational green space system layout based on fractal quantification model from the perspective of urban-rural integration," Landscape Architecture, vol. 25, no. 12, pp. 81-86, 2018.

[29] J. Liu, L. Zhang, Y. W. Ji, and Q. P. Zhang, "Spatial-temporal evolution analysis of urban green space system based on fractal model: a case study of downtown Shanghai," Modern Urban Research, vol. 10, pp. 12-19, 2019.

[30] P.-A. Versini, A. Gires, I. Tchiguirinskaia, and D. Schertzer, "Fractal analysis of green roof spatial implementation in European cities," Urban Forestry \& Urban Greening, vol. 49, Article ID 126629, 2020.

[31] Y. G. Chen, "Approaches to estimating fractal dimension and identifying fractals of urban form," Progress in Geography, vol. 36, no. 5, pp. 529-539, 2017.

[32] Y.-Q. Long and Y.-G. Chen, "Fractal characterization of structural evolution of Beijing, Tianjin and Hebei transportation network," Human Geography, vol. 34, no. 4, pp. 115-125, 2019.

[33] F. Zhang, Y. G. Chen, and X. S. Li, "Radial dimension analysis of growth and form of cities in the Beijing-Tianjin-Hebei region," Progress in Geography, vol. 38, no. 1, pp. 65-76, 2019.

[34] M. Batty and P. Longley, Fractal Cities: A Geometry of Form and Function, Academic Press, Cambridge, MA, USA, 1994.

[35] S.-G. Jiang and Y.-X. Zhou, "The fractal urban form of Beijing and its practical significance," Geographical Research, vol. 25, no. 2, pp. 204-212, 2006. 